\title{
RADIOLOGY OF HAND-MIRROR OF VARIOUS SYSTEMIC DISEASES
}

Sadashiva Gowda $H^{1}$

${ }^{1}$ Associate Professor, Department of Radiodiagnosis, Vijaynagar Institute of Medical Sciences, Bellary.

\section{ABSTRACT}

Modern diagnostic imaging offers a vast spectrum of sophisticated modalities and techniques, which enables us to study the function and morphology of the human body. However, even in the most advanced imaging department in the economically privileged parts of the world, $70-80 \%$ of all clinically relevant questions may be solved by using the two main cornerstones of diagnostic imaging, which are radiography and ultrasonography.

\section{KEYWORDS}

Systemic Diseases, Osteopetrosis, Pyknodysostosis.

HOW TO CITE THIS ARTICLE: Sadashiva Gowda H. Radiology of hand-mirror of various systemic diseases. J. Evolution Med. Dent. Sci. 2016;5(8):366-370, DOI: 10.14260/jemds/2016/83

\section{INTRODUCTION}

Modern diagnostic imaging offers a vast spectrum of sophisticated modalities and techniques, which enables us to study the function and morphology of the human body. However, even in the most advanced imaging department in the economically privileged parts of the world, $70-80 \%$ of all clinically relevant questions may be solved by using the two main cornerstones of diagnostic imaging, which are radiography and ultrasonography.

Several systemic diseases manifest on the radiographs of hand. Conventional radiographs of hand are excellent source of information in most of the systemic diseases. Sophisticated imaging modalities may not be necessary for definitive diagnoses. We bring to you a pictorial essay showing some diseases manifesting on radiographs of hand.

\section{DISCUSSION}

\section{A. Congenital/Developmental. 1,2 Osteopetrosis}

Osteopetrosis is a rare hereditary and familial bone abnormality characterized by defective osteoclast function. ${ }^{[1]}$ Bone remodelling is impaired and the primary calcified spongiosa cannot be effectively reabsorbed resulting in sclerotic bones, which are thick but structurally weak and brittle. Normal bone may be laid down in episodes, so that zones of normal and of denser bone may be seen. Marrow is encroached upon and extra-medullary hematopoiesis occurs. ${ }^{[2]}$ The radiological findings depend upon the severity of disease. There is generalized increase in bone density with obliteration of normal trabecular pattern and little or no differentiation between cortical and medullary portions. Bone in bone appearance or endobones may also be seen. Longitudinal metaphyseal striations are also seen.[1] are stubby with spoon-shaped nails (koilonychias) and finger clubbing. Hypoplasia/absence of the lateral ends of clavicles and terminal tufts of the fingers and toes (Acro-osteolysis) is a consistent finding.[2]

Financial or Other, Competing Interest: None.

Submission 02-08-2015, Peer Review 22-08-2015,

Acceptance 28-08-2015, Published 28-01-2016.

Corresponding Author:

Dr. Sadashiva Gowda $H$,

Associate Professor,

Department of Radiodiagnosis,

VIMS, Bellary, Karnataka.

E-mail: drsadashiv27@yahoo.com

DOI: $10.14260 /$ jemds $/ 2016 / 83$
The long bones have elongated and flared metaphyses. Involvement of hands and feet parallel changes in the long bones.

\section{Pyknodysostosis}

Pyknodysostosis is a rare autosomal recessive disorder characterized by increased bone density with preservation of medullary canals, dwarfism and skeletal fragility.[2] the facial appearance is characteristic with a beaked nose, prognathic jaw, small face and a prominent forehead. The hands and feet

\section{Osteopoikilosis}

Osteopoikilosis is an autosomal dominant disorder characterized by small round or ovoid radio-opacities appearing in the juxta-articular regions of bone. The majority of the cases are asymptomatic and found on radiographic studies taken for other reasons. Although, it can be seen at any age.[1] it is rarely detected before age 3 . Males are affected more frequently than females.

Multiple small radio-opacities found scattered in epiphyseal and metaphyseal regions are generally pathognomonic. The lesions are symmetric with a predilection for the long tubular bones, carpals and tarsals. Generally, the size ranges from 1 to $10 \mathrm{~mm}$ in diameter.

\section{Marfan's Syndrome}

Marfan's syndrome is an autosomal dominant entity consisting of long, slender, tubular bones, ocular abnormalities and aortic aneurysm. Majority of patients have familial incidence. A defect on chromosome 15 is responsible for clinical manifestations of Marfan's syndrome.[1] The clinical manifestations defining Marfan's syndrome involve three systems: skeletal, ocular and cardiovascular. The elongation of tubular bones (The trunk is spared) is most marked in distal portion of the extremities, especially the phalanges, metacarpals and metatarsals. Generally, affected individuals are taller than 6 feet. Classically, the skull reveals dolichocephaly.[1] Elongation of the extremities without an increase in width is characteristic. The tubular bones of the hands and feet are particularly long, slender and gracile; hence the term arachnodactyly or spider-like fingers. Radiographically, the metacarpal index (The ratio of length to width of the second through fifth metacarpal bones) is increased. 


\section{Pseudoachondroplasia}

Pseudoachondroplasia is inherited in an autosomal dominant manner. It is a form of short-limb dwarfism. Often the diagnosis is not made at birth, but when the child is 2-3 years of age.[1] at first growth is normal. At 2-3 years of age, the diagnosis is suspected because of a delay in walking or an unusual waddling gait is noted. As the growth rate slows, the trunk appears long with lumbar lordosis and rhizomelic shortening of the limbs.

Final adult height is $80-130 \mathrm{~cm}$. Individuals with Pseudoachondroplasia have normal head size and facial features. Radiographic changes include shortening of tubular bones, flaring of metaphyses, variable epiphyseal maldevelopment and some degree of vertebral end-plate malformation.

\section{Metaphyseal Dysplasia/Pyle's disease}

Metaphyseal dysplasia is a rare autosomal recessive disorder characterized by failure of metaphyseal remodeling resulting in splaying or flaring of ends of long bones (Erlenmeyer flask deformity). It manifests at variable ages, most commonly in late childhood.[2] It is a benign disorder with unimpaired general health and a normal lifespan. Many patients are taller than normal, presenting with bulbous enlargement of the lower extremity joints. The most commonly involved bones are the distal femur, tibia (Proximal and distal) and proximal fibula. In the upper extremity involvement of the distal radius, ulna and proximal humerus is most frequent. Occasionally, the hands show metaphyseal flaring in small tubular bones.

\section{Hereditary Multiple Exostosis/Diaphyseal Aclasis}

Is an inherited autosomal dominant metaphyseal overgrowth that is characterized by multiple osteochondromas.[2] It is most commonly discovered between 2 to 10 years and both sexes are equally affected. Patient presents with painless, lumpy joints. The metaphyseal portions of long bones are commonly affected. Distribution is bilateral and symmetric. Hands are involved in advanced cases.

\section{B. Infective/Inflammatory Disorders. ${ }^{2}$ \\ Tubercular Dactylitis/Spina Ventosa}

Tubercular dactylitis is a rare unusual presentation of tuberculosis occurring in short tubular bones of the hands and feet. It is most commonly seen in children less than 5 years of age. It is characterized by multiple lesions of consecutive onset. Monostotic involvement is common. The typical lesion causes diffuse soft tissue swelling, bone expansion and thinning of cortex.

\section{Gout}

Gout is a disorder of purine metabolism in which hyperuricemia leads to deposition of sodium monourate crystals into cartilage, synovium, periarticular and subcutaneous tissues. These crystals evoke a strong inflammatory arthritis. It affects males usually in the $4^{\text {th }}$ and $5^{\text {th }}$ decades. The disease has four stages: asymptomatic hyperuricemia, acute gouty arthritis, polyarticular gouty arthritis (Chronic, long-standing disease) and chronic tophaceous gout (soft tissue accumulation of sodium monourate). General radiologic features include dense soft tissue tophi, preservation of joint space, bone erosions
(Marginal, periarticular overhanging margin sign, intraosseous) normal bone density, periosteal new bone, secondary degenerative joint changes, chondrocalcinosis and avascular necrosis. Radiograph of hand shows asymmetric joint involvement of any articulation. Erosions, soft tissue swelling and misalignments are the main features. There may be erosions of the carpus (Spotty carpal sign) and ulnar styloid process. Bone density is normal.

\section{Leprosy}

Leprosy commonly involves the bones of hand with osteolytic lesions and marked resorption of bones. Super-imposed infection is often present producing osteomyelitic features.

\section{Endocrinal and nutritional/metabolic disorders.3,4 Acromegaly}

In adulthood, excessive growth hormone secretion from a pituitary eosinophilic adenoma produces growth of intramembranous bone tissue and subcutaneous hypertrophy. This is especially prominent in hands and feet (Acral parts) from which the term acromegaly is derived. Heel pad thickness is greater than $20 \mathrm{~mm}$. Skull radiograph shows sella turcica enlargement, sinus overgrowth and malocclusion. Hand radiograph shows widened shafts of phalanges and metacarpals, bony protuberances and prominent ungula tufts, which may assume a spade-like appearance. A reliable indicator is the generalized increase in joint space width owing to cartilage overgrowth.

\section{Hypothyroidism (Cretinism)}

Hypothyroidism or cretinism causes retarded bone age with characteristic stippled epiphyses.

\section{Hyperparathyroidism}

Hyperparathyroidism is the general term applied to overactivity of the parathyroid gland. Increased parathyroid activity liberates parathormone, which exerts a strong osteoclastic effect on the skeleton. It affects females $3: 1$, usually in the age group of 30-50 years who present with weakness, lethargy, polydipsia and polyuria.

Bone changes consist of osteopenia, accentuated trabecular patterns, subperiosteal resorption, loss of cortical definition and brown tumors. Soft tissue changes include nephrocalcinosis, renal calculi, chondrocalcinosis and calcification in various periarticular tissues and visceral organs. Hand radiograph shows characteristic subperiosteal resorption located at the second and third digits, proximal and middle phalanges, especially on their radial margins. Additional features include acro-osteolysis.

\section{Miscellaneous. 4,5}

\section{Hypertrophic Osteoarthropathy}

The disease is composed of a triad of digital clubbing, symmetric arthritis and periostitis occurring as a sequel to major visceral disorder, usually intrathoracic in location. Most common in males in $4^{\text {th }}-6^{\text {th }}$ decades. Radiographs show bilateral, symmetric, linear periostitis (most common in the tibia, fibula, radius and ulna), joint effusions, digital clubbing visible as a bulbous enlargement of the distal fingertips. 


\section{Tumoral Calcinosis}

Tumoral calcinosis is a disorder of unknown cause characterized by localized accumulations of calcific masses. Commonly affects children and adolescents of 6-25 years. Blacks are more commonly involved. Patient presents with painless, enlarging periarticular soft tissue masses. Radiographs show dense, lobulated, well-marginated soft tissue masses with normal adjacent bones and joints.

\section{Lobster Claw Hand. ${ }^{5}$}

It is a rare anomaly. Phalanges of middle finger and sometimes corresponding metacarpal are absent and cleft in the plane of the missing digit and metacarpal separates the hand into medial and lateral portions. Digits on either side of cleft may exhibit various degrees of fusion, in many cases is associated with tibial hemimelia.

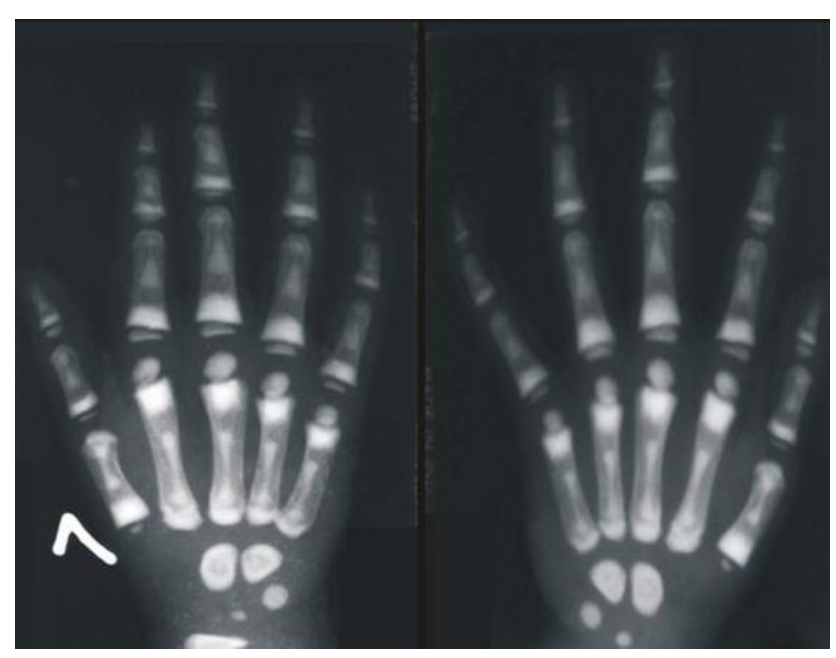

Fig. 1: Increased densities within the metacarpals and phalanges showing a bone within a bone appearance

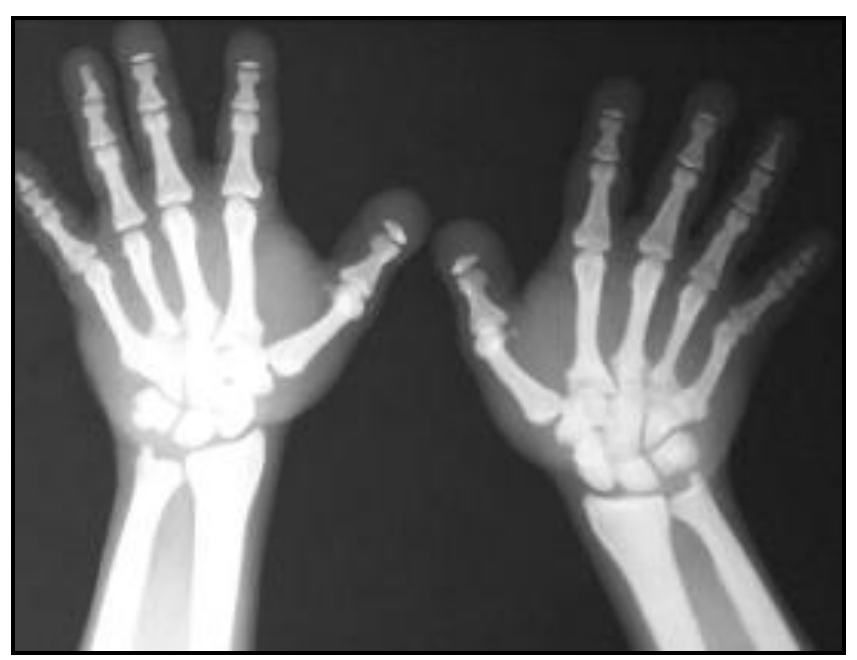

Fig. 2: Radiograph shows generalized increase in bone density with preservation of medullary canals and acro-osteolysis

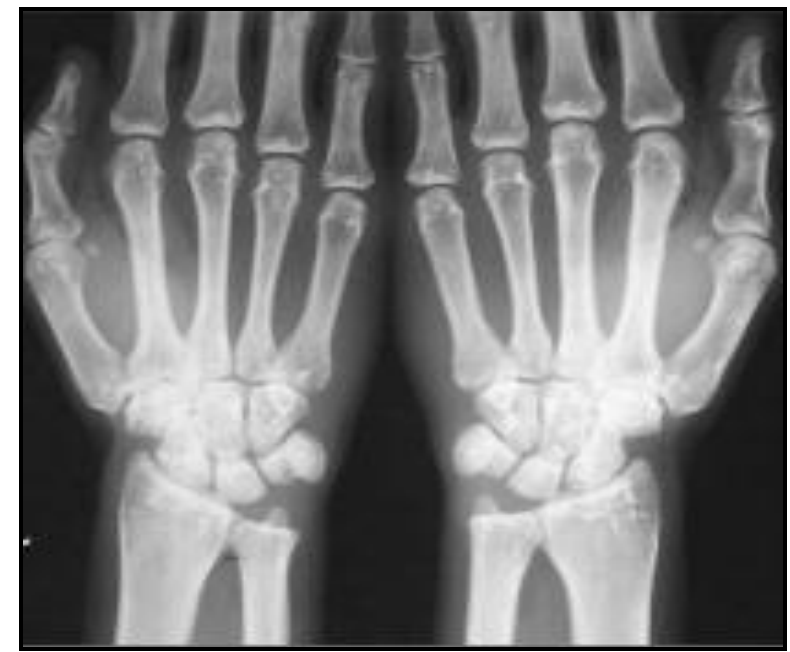

Fig. 3: Radiograph showing round/oval radiopacities in the juxta-articular regions of the visualized bone

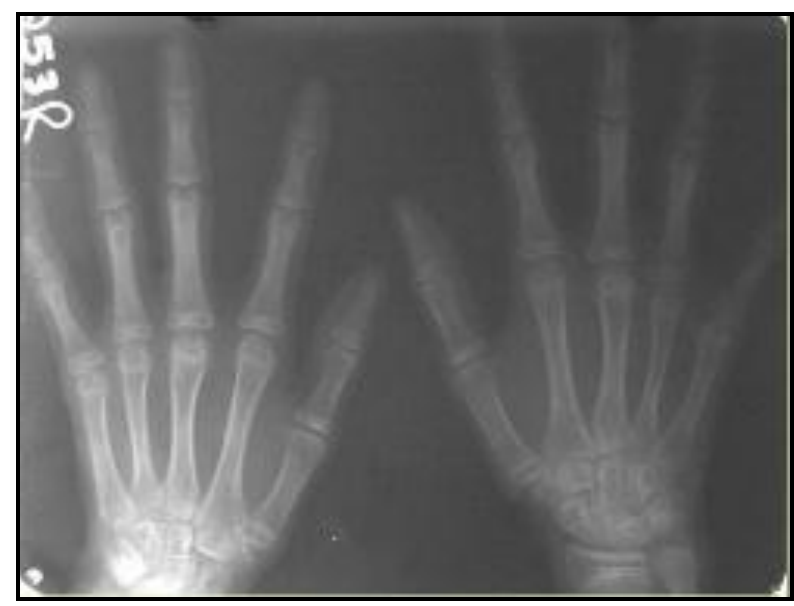

Fig. 4: The tubular bones of the hands (Metacarpals and phalanges) are long, slender and gracile - Arachnodactyly or spider-like fingers

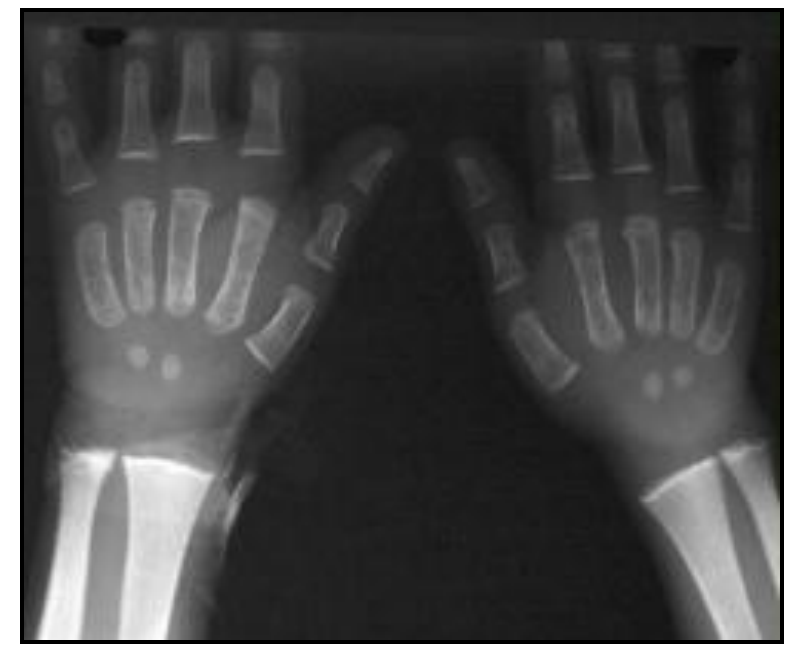

Fig. 5: The distal radius and ulna, phalanges and metacarpals show flared irregular metaphyses. Phalanges and metacarpals are short. There is proximal pointing of 2nd to 5th metacarpal 


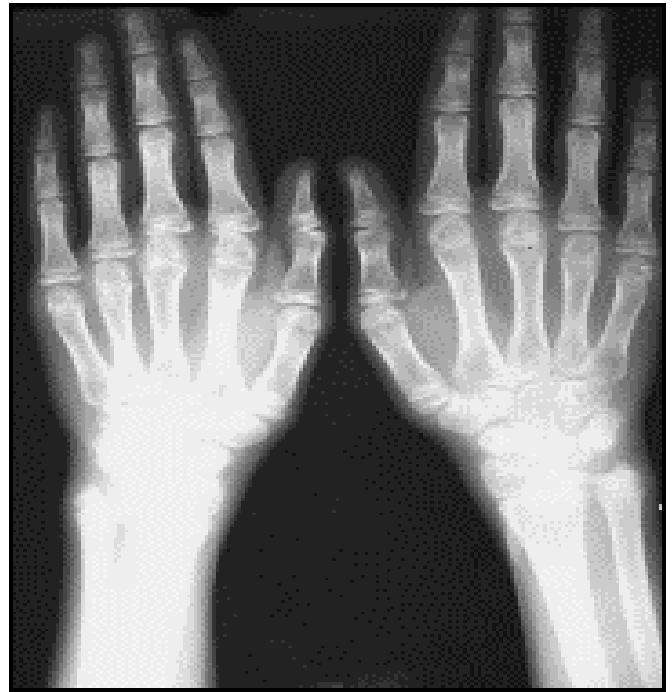

Fig. 6: PA film shows ulnar slant of the distal articular surface of radius due to premature closure of medial portion of the distal radial epiphysis. Distal radioulnar joint is widened. Interosseous space is increased. Lateral film shows volar tilt of distal radial articular surface and posterior subluxation of lower ulna. Hand and carpus project forward at the wrist joint to produce a bayonet-like appearance

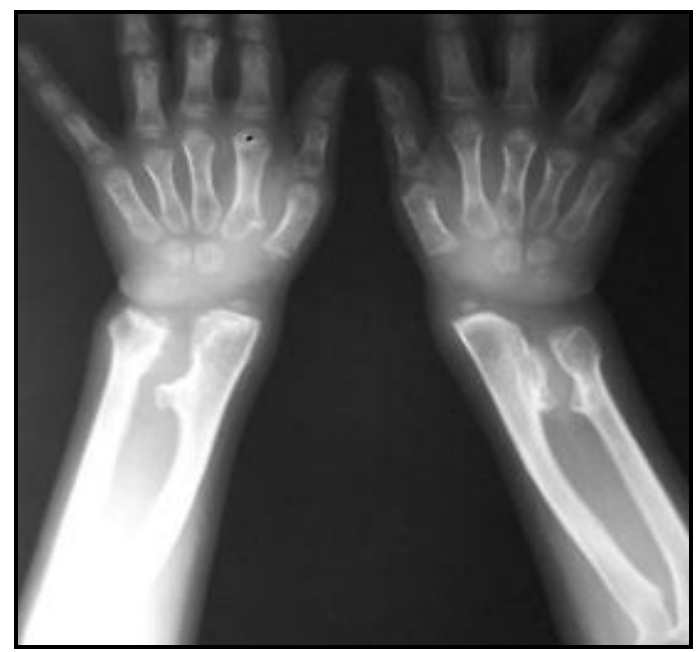

Fig. 7: Multiple bony outgrowths seen in distal ends of right radius and ulna, Distal end of left radius and proximal end of left 2nd metacarpal. Ulna is shortened, Radius shows outward bowing. Radioulnar joint is subluxated

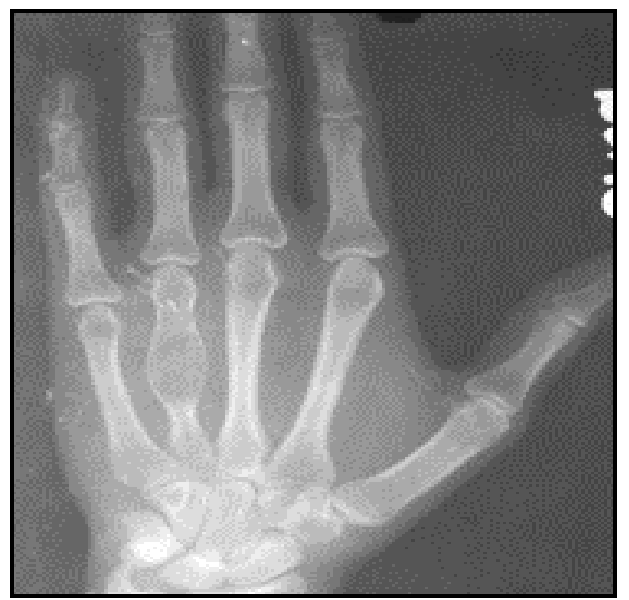

Fig. 8: Radiograph showing medullary expansion of the shaft of the $4^{\text {th }}$ metacarpal of the left hand with thinning of the overlying cortex

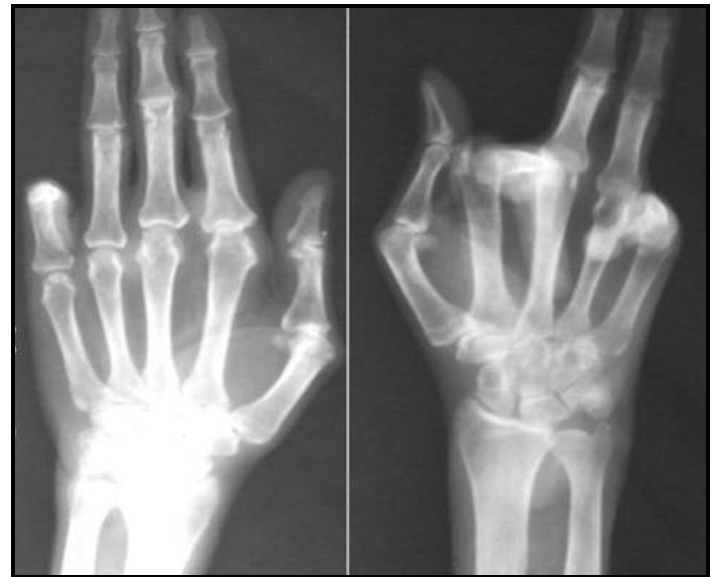

Fig. 9: Radiograph showing marginal erosions around the MCP and PIP joints of the left index finger. Bone density is normal and joint spaces are well preserved

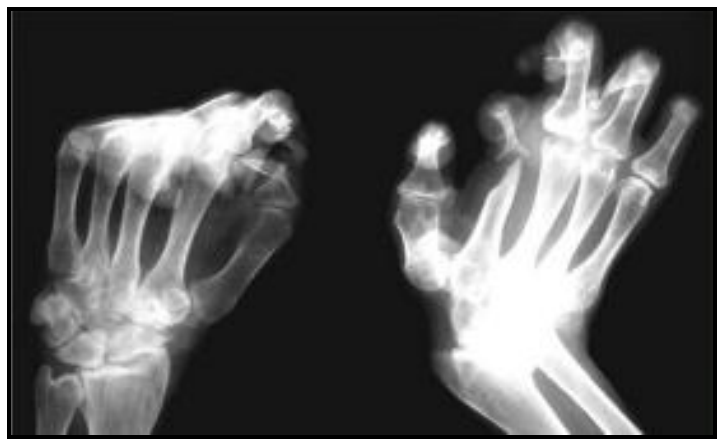

Fig. 10: Radiograph showing eccentric absorption of distal second metacarpal of right hand (Licked-candy stick configuration), cupping of right $2^{\text {nd }}$ metacarpophalangeal joint (Cup and pencil appearance) and flexion deformity of the fingers (Claw hand). Bones are osteoporotic

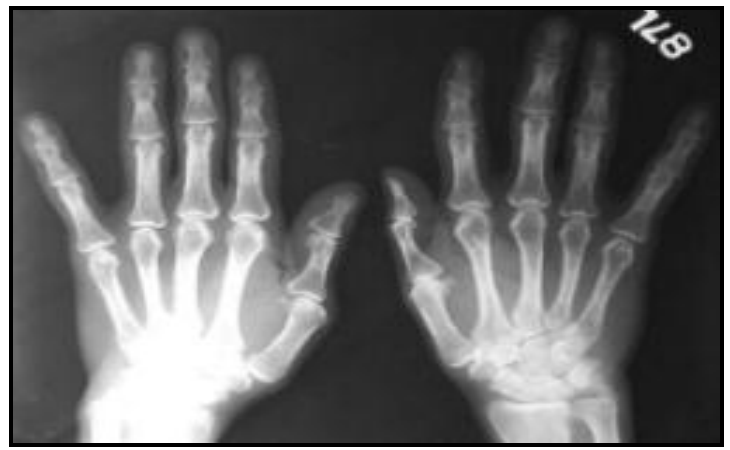

Fig. 11: Radiograph shows enlargement of phalanges and soft tissues and spade like terminal tufts

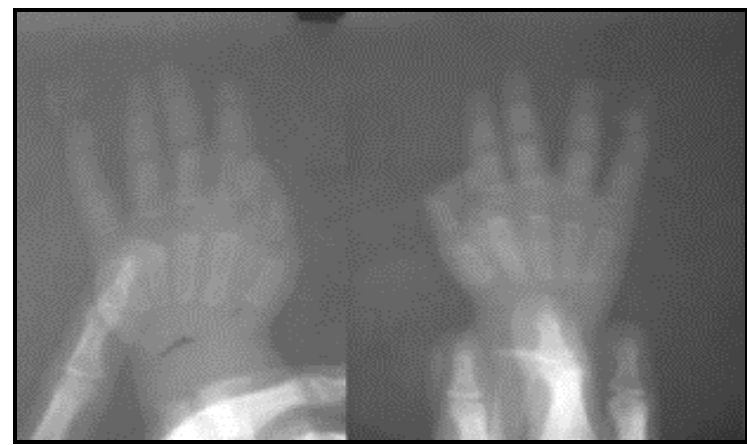

Fig. 12: Radiograph showing retarded skeletal growth and maturation. There is delayed appearance of ossification centres of carpal bones and epiphyseal dysgenesis 


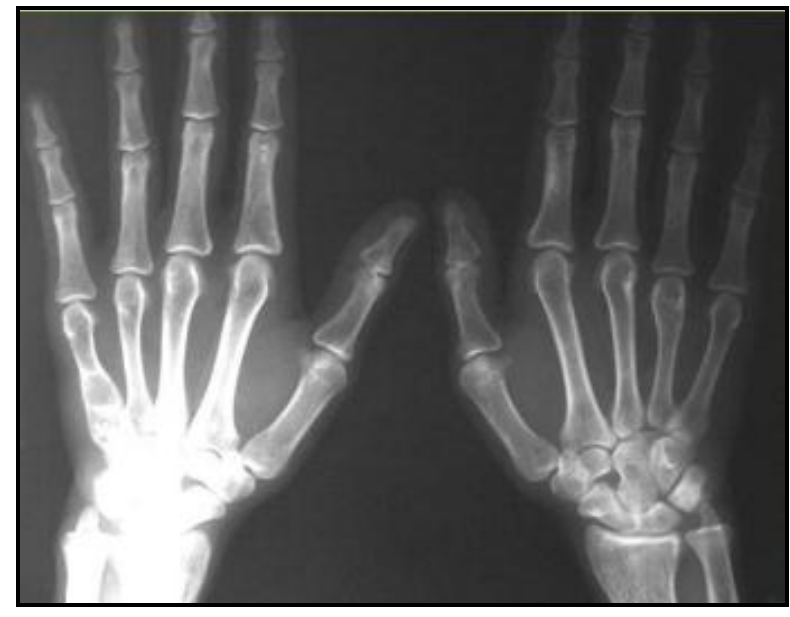

Fig. 13: Radiograph showing subperiosteal resorption of the radial margins of the proximal and middle phalanges of the second and third digits. Central, slightly expansile, septated,

lytic lesion is seen in proximal end of the fifth metacarpal (Brown tumour)

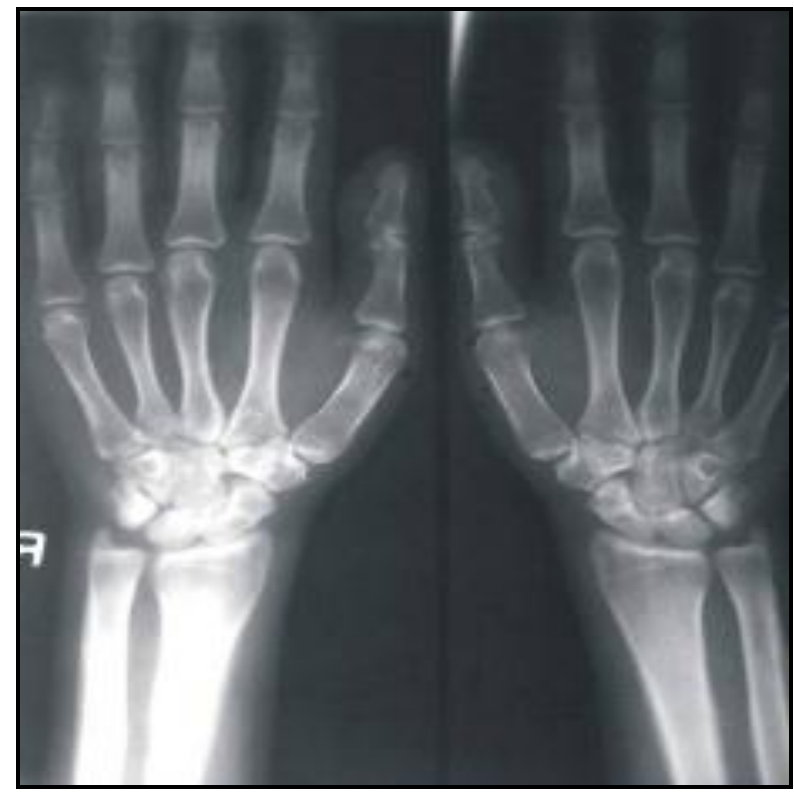

Fig. 14: Radiograph showing bilateral, symmetric, linear periostitis involving the lower one-third of radius and ulna

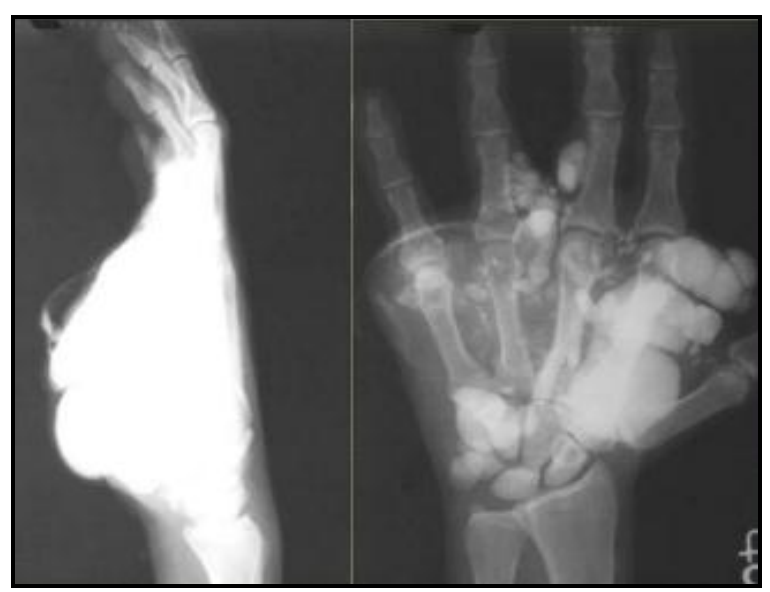

Fig. 15: Radiograph showing dense, lobulated, wellmarginated peri-articular soft tissue masses with calcification. Adjacent bones and joints are normal

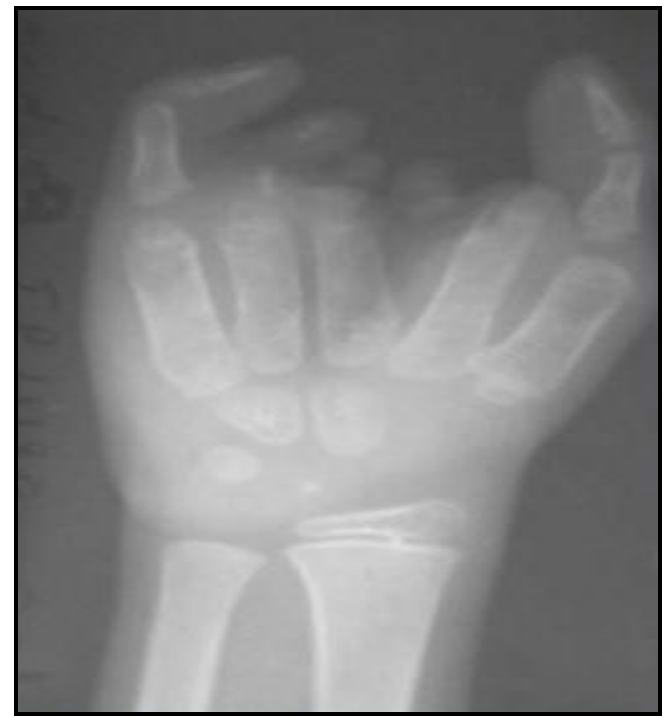

Fig. 16: Radiograph showing hypoplasia of phalanges of index, middle and ring fingers and a cleft in the plane of missing digits

\section{REFERENCES}

1. Peter Renton. In: Sutton D, ed. In: textbook of radiology and imaging, $6^{\text {th }}$ edn. China: Churchill Livingstone, 2003;1107-1171.

2. Wilson DJ, Berendt AR. In: Grainger RG, Allison, Adam A, Dixon AK, eds. Diagnostic Radiology- A Textbook of Medical Imaging, 4th edn, China: Churchill Livingstone, 2001;2057-2072.

3. Adam Greenspan. In: orthopaedic imaging -a practical approach, 4th edn, Philadelphia: Williams and Wilkins, 2004;859-871.

4. Yochum TR, Rowe LJ. In: essentials of skeletal radiology, 3rd edn, USA: Williams and Wilkinsons, 2005;1080-1084.

5. Lane NE, Nevitt MC, Genant HK, et al. Reliability of new indices of radiographic osteoarthritis of the hand and hip and lumbar disc degeneration. J Rheumatol 1994;20(11):1911-8. 